\title{
Impact of Mobile Event Movement on the Performance of Wireless Sensor Networks
}

\author{
Tao Yang*, Leonard Barolli ${ }^{\dagger}$, Gjergji Mino ${ }^{\ddagger}$, Fatos Xhafa ${ }^{\S}$ and Arjan Durresi ${ }^{\Uparrow}$ \\ * JSPS Fellow Researcher \\ Fukuoka Institute of Technology (FIT) \\ 3-30-1 Wajiro-Higashi, Higashi-Ku, Fukuoka 811-0295, Japan \\ E-mail: taoyang.tou@gmail.com \\ ${ }^{\dagger}$ Department of Information and Communication Engineering \\ Fukuoka Institute of Technology (FIT) \\ 3-30-1 Wajiro-Higashi, Higashi-Ku, Fukuoka 811-0295, Japan \\ Email: barolli@fit.ac.jp \\ ${ }^{\ddagger}$ Graduate School of Engineering \\ Fukuoka Institute of Technology (FIT) \\ 3-30-1 Wajiro-Higashi, Higashi-Ku, Fukuoka 811-0295, Japan \\ Email: bd09002@bene.fit.ac.jp \\ $\S$ Technical University of Catalonia \\ Department of Languages and Informatics Systems \\ C/Jordi Girona 1-3, 08034 Barcelona, Spain \\ E-mail:fatos@lsi.upc.edu \\ "Department of Computer and Information Science \\ Indiana University Purdue University at Indianapolis (IUPUI) \\ 723 W. Michigan Street SL 280 Indianapolis, IN 46202, USA \\ durresi@cs.iupui.edu
}

\begin{abstract}
In this paper, we investigate how the sensor network performs in the case when the event moves with special movement path. We compare the simulation results for four scenarios: when the event is stationary, moving randomly, moving with simple 4 path and boids path. The simulation results have shown that for the case when event is moving randomly the performance is the worst in the four scenarios. The characteristic of goodput decreases with the increase of number of sensor nodes. In the case of boids model, the gooput is unstable when the $T_{r}$ is lower than 10 pps. The consumed energy characteristic increases with the increase of $T_{r}$. Simulation results show that the consumed energy of random movement is the worst among four scenarios. The consumed energy of boids model is the lowest in four cases. This show that the event movement with boids model can decrease the consumed energy in the large scale WSNs.
\end{abstract}

Keywords-WSN, Event mobility, Movement path, Goodput, Consumed energy, Radio model.

\section{INTRODUCTION}

In recent years, technological advances have lead to the emergence of distributed Wireless Sensor Networks (WSNs) which are capable of observing the physical world, processing the data, making decisions based on the observations and performing appropriate actions. These networks can be an integral part of systems such as battle-field surveillance and micro-climate control in buildings, nuclear, biological and chemical attack detection, home automation and environmental monitoring [1], [2].

Wireless sensor network simulation is an important part of the current research. A large number of algorithms were first implemented and evaluated using several network sim- ulators. Recently, there are many research works for sensor networks [3], [4]. In our previous work [5], we implemented a simulation system for sensor networks consider different protocols (e.g., AODV, DSR, DSDV, OLSR) and different propagation radio models. In [6], we analysed the performance of the WSNs considering different topologies with the irregular radio model. Also, we analysed the performance of our proposed simulation systems. But, we considered that the event node is stationary in the observation field. However, in many applications the event node may move. For example, in an ecology environment the animals may move randomly. Another example is when an event happens in a robot or in a car.

In this work, we want to investigate how the sensor network performs in the case when the event moves with special movement path. We carry out simulations for lattice topology and TwoRayGround radio model considering Adhoc On-demand Distance Vector (AODV) protocol. We evaluate the performance of WSN for 4 scenarios: when the event is stationary, moving randomly, moving with simple 4 path and boids path. The simulation results have shown that for the case when event is moving randomly the performance is the worst in the four scenarios. The characteristic of goodput decreases with the increase of number of sensor nodes. In the case of boids model, the gooput is unstable when the $T_{r}$ is lower than $10 \mathrm{pps}$. The consumed energy characteristic increases with the increase of $T_{r}$. Simulation results show that the consumed energy of random movement is the worst among four scenarios. 


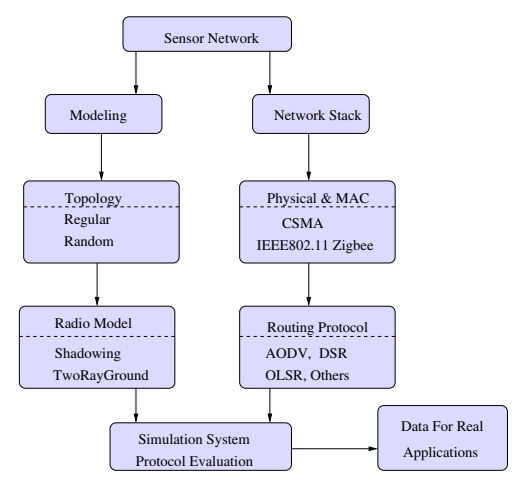

Figure 1. Proposed network simulation model.

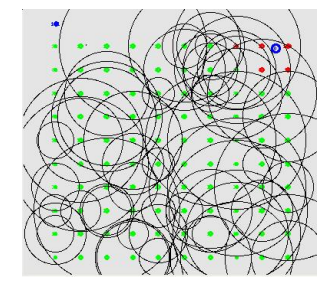

Figure 2. An example of lattice network.

The remainder of the paper is organized as follows. In Section II, we explain the proposed network simulation model. In Section III, we discuss the movement path of event. In Section IV, we show the simulation results. Conclusions of the paper are given in Section V.

\section{Proposed Network Simulation Model}

In our WSN, every node detects the physical phenomenon and sends back to the sink node the data packets. We suppose that the sink node is more powerful than sensor nodes. In our previous work, the event node was stationary. In this work, we consider that the event moves with special movement path. We analyse the performance of the network in a fixed time interval. This is the available time for the detection of the phenomenon and its value is application dependent. Proposed network simulation model is shown in Fig.1.

\section{A. Topology}

For the physical layout of the WSN, two types of topologies has been studied so far: random and lattice topologies. In the former, nodes are supposed to be uniformly distributed, while in the latter one nodes are vertexes of particular geometric shape, e.g., a square grid. For lattice topology, in order to guarantee the connectedness of the network we should set the transmission range of every node to the step size, $d$, which is the minimum distance between two rows (or columns) of the grid [7], [8]. In fact, by this way the number of links that every node can establish (the node degree $\mathrm{D}$ ) is 4 . Nodes at the borders have $D=2$.

In the case of random networks, we suppose that the coordinates in the Euclidean plane of every sensor are random variables uniformly distributed in the interval $[0, L] \times[0, L]$. Snapshots of lattice and random networks generated in simulations are shown in Fig. 2 and Fig. 3, respectively.

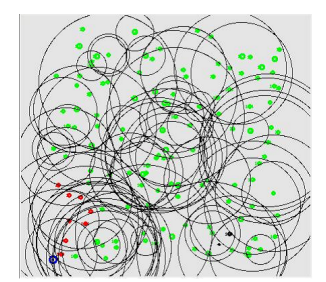

Figure 3. An example of random network.

\section{B. Radio Model}

In order to simulate the detection of a natural event, we used the libraries from Naval Research Laboratory (NRL) [9]. In this framework, a phenomenon is modelled as a wireless mobile node. The phenomenon node broadcasts packets with a tunable synchrony or pulse rate, which represents the period of occurrence of a generic event. These libraries provide the sensor node with an alarm variable. The alarm variable is a timer variable. It turns off the sensor if no event is sensed within an alarm interval. In addition to the sensing capabilities, every sensor can establish a multi-hop communication towards the sink by means of a particular routing protocol.

We assume that the MAC protocol is the IEEE 802.11 standard. This serves to us as a baseline of comparison for other contention resolution protocols. The receiver of every sensor node is supposed to receive correctly data bits if the received power exceeds the receiver threshold, $\gamma$.

As reference, we select parameters values according to the features of a commercial device (MICA2 OEM). In particular, for this device, we found that for a carrier frequency of $f=916 \mathrm{MHz}$ and a data rate of $34 \mathrm{KBaud}$, we have a threshold (or receiver sensitivity) $\left.\gamma\right|_{d B}=-118 \mathrm{dBm}$ [10]. The calculation of the phenomenon range is not yet optimized and the phenomenon propagation is assumed to follow the propagation laws of the radio signals.

In particular, the emitted power of the phenomenon is calculated according to a TwoRayGround propagation model [11]. The received power $P_{r}$ at a certain distance $d$ is the same along all directions in the plane. In the case of Line Of Sight (LOS) propagation of the signal, the Friis formula predicts the received power as:

$$
\begin{aligned}
& P_{r}(d)=P_{t}-\beta(\mathrm{dB}), \\
& \beta=10 \log \left(\frac{(4 \pi d)^{2} L}{G_{t} G_{r} \lambda^{2}}\right)
\end{aligned}
$$

where $G_{r}$ and $G_{t}$ are the antenna gains of the receiver and the transmitter, respectively, $\lambda$ is the wavelength of the signal, $L$ the insertion loss caused by feeding circuitry of the antenna, and $\beta$ is the propagation pathloss. For omni-antennas, $G_{R}=G_{t}=1$. The signal decay is then proportional to $d^{2}$. A more accurate model is Two-RayGround model, where in addition to the direct ray from the transmitter towards the receiver node, a ground reflected signal is supposed to be present. Accordingly, the received 
power depends also on the antenna heights and the pathloss is:

$$
\beta=10 \log \left(\frac{(4 \pi d)^{4} L}{G_{t} G_{r} h_{t} h_{r} \lambda^{2}}\right)
$$

where $h_{r}$ and $h_{t}$ are the receiver and transmitter antenna heights, respectively. The power decreases faster than Eq. (1). The formula in Eq. (2) is valid for distances $d>d_{c}\left(d_{c}\right.$ is the distance threshold of signal LOS propagation), that is far from the transmitting node [12].

\section{Energy Model}

The energy model concerns the dynamics of energy consumption of the sensor. A widely used model is as follows [13]. When the sensor transmits $k$ bits, the radio circuitry consumes an energy of $k P_{T x} T_{B}$, where $P_{T x}$ is the power required to transmit a bit which lasts $T_{B}$ seconds. By adding the radiated power $P_{t}(d)$, we have:

$$
E_{T x}(k, d)=k T_{B}\left(P_{T x}+P_{t}(d)\right) .
$$

Since packet reception consumes energy, by following the same reasoning, we have:

$$
E(k, d)=k P_{T x} T_{B}+k T_{B} P_{t}(d)+k P_{R x} T_{B}
$$

where $P_{R x}$ is the power required to correctly receive (demodulate and decode) one bit.

\section{Routing Protocols}

We are aware of many routing protocols for ad-hoc networks [14]. We have implemented in our simulation system many routing protocols. But, in this work, we consider only AODV protocol.

The AODV is an improvement of DSDV to on-demand scheme. It minimize the broadcast packet by creating route only when needed. Every node in network maintains the route information table and participate in routing table exchange. When source node wants to send data to the destination node, it first initiates route discovery process. In this process, source node broadcasts Route Request (RREQ) packet to its neighbors. Neighbor nodes which receive RREQ forward the packet to its neighbor nodes. This process continues until RREQ reach to the destination or the node who know the path to destination.

When the intermediate nodes receive RREQ, they record in their tables the address of neighbors, thereby establishing a reverse path. When the node which knows the path to destination or destination node itself receive RREQ, it send back Route Reply (RREP) packet to source node. This RREP packet is transmitted by using reverse path. When the source node receives RREP packet, it can know the path to destination node and it stores the discovered path information in its route table. This is the end of route discovery process. Then, AODV performs route maintenance process. In route maintenance process, each node periodically transmits a Hello message to detect link breakage.

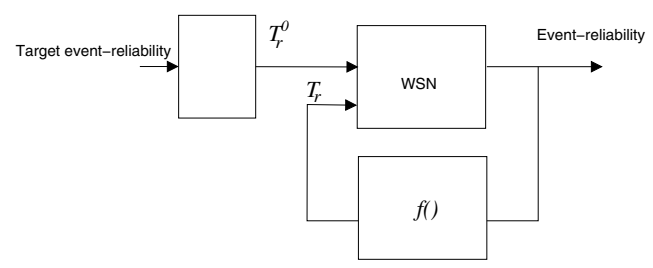

Figure 4. Representation of the transport based on the event-reliability.

\section{E. Event Detection and Transport}

For event detection and transport, we use the data-centric model similar to [15], where the end-to-end reliability is transformed into a bounded signal distortion concept. In this model, after sensing an event, every sensor node sends sensed data towards the Monitoring Node (MN). The transport used is a UDP-like transport, i.e. there is not any guarantee on the data delivery. While this approach reduces the complexity of the transport protocol and well fit the energy and computational constraints of sensor nodes, the event-reliability can be guaranteed to some extent because of the spatial redundancy.

The sensor node transmits data packets reporting the details of the detected event at a certain transmission rate. The setting of this parameter, $T_{r}$, depends on several factors, as the quantization step of sensors, the type of phenomenon, and the desired level of distortion perceived at the MN. For example, if we refer to event-reliability as the minimum number of packets required at sink in order to reliably detect the event, then whenever the sink receives a number of packets less than the event-reliability, it can instruct sensor nodes to use a higher $T_{r}$. This instruction is piggy-backed in dedicated packets from the MN.

This system can be considered as a control system, as shown in Fig. 4, with the target event-reliability as input variable and the actual event-reliability as output parameter. The target event-reliability is transformed into an initial $T_{r}^{0}$. The control loop has the output event-reliability as input, and on the basis of a particular non-linear function $f(\cdot), T_{r}$ is accordingly changed. We do not implement the entire control system, but only a simplified version of it. For instance, we vary $T_{r}$ and observe the behaviour of the system in terms of the mean number of received packets. In other words, we open the control loop and analyse the forward chain only.

\section{Event Mobility IN MANY-TO-ONE MULTI-HOP WSNS}

The research works on the subject of event mobility in many-to-one multi-hop WSNs can generally be categorized based on the assumed type of event trajectory. Types of mobile event trajectories most commonly studied in the literature include: fixed (stationary), random, 4 path and boids path model. We explain each of these four types of event trajectories in more details in following. 


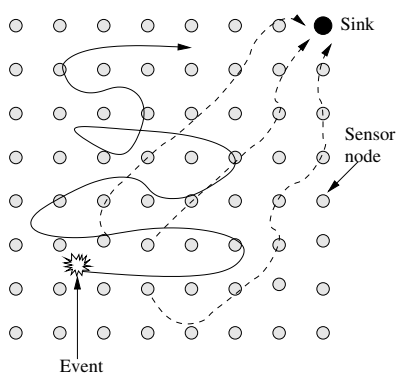

Figure 5. Multi-hop WSN with a randomly moving event.

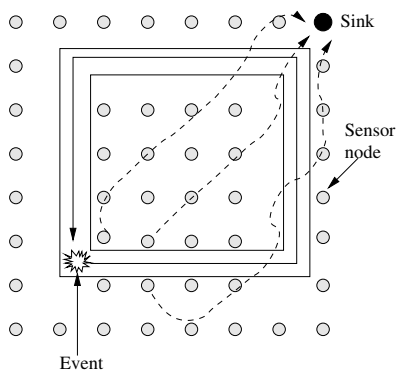

Figure 6. A simple 4 path-constrained mobile event.

\section{A. Stationary Event Model}

In general, the event is stationary in the observed field (for e.g., gas, fire). Sensor nodes which are around the event sense the event and send the information to the sink by multihop.

\section{B. Movement with Random Path}

As the name implies, the trajectory of a randomly moving event comprises a sequence of segments of arbitrary length and direction (see Fig. 5). The event's speed along each segment, and the pause time between movements along different segments, can also be arbitrary, although these two conditions do not to hold to satisfy the randomness requirement. In many applications the event node may move. For example, in an ecology environment the animals may move randomly. Another example is when an event happens in a robot or in a car.

\section{The Simple 4 Path Model}

In contrast to the random trajectory, the fixed trajectory is fully deterministic and the event is expected to continuously follow the same path through the network. The fixed trajectory is typically forced upon the mobile event by the nature of the physical terrain and/or presence of obstacles in the environment. An example of a research study that deals with this type of trajectory is [16]. In this work, we set the mobile event with simple 4 path (see Fig. 6).

\section{The Boids Path Model}

As computer model of coordinated animal motion such as bird flocks and fish schools. It was based on three dimensional computational geometry of the sort normally used in computer animation or computer aided design. So called

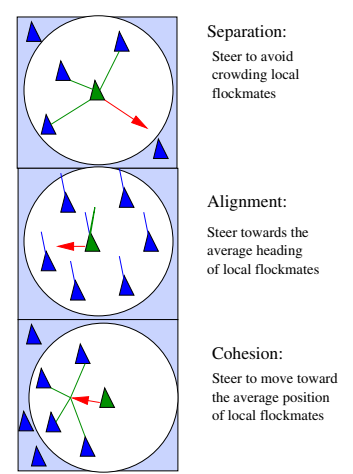

Figure 7. Boids model.

Table I

TOPOLOGY SETTINGS.

\begin{tabular}{|c|c|}
\hline \multicolumn{2}{|c|}{ Lattice } \\
\hline \hline Step & $d=\frac{L}{\sqrt{N}-1} \mathrm{~m}$ \\
\hline Service Area Size & $L^{2}=(800 \times 800) \mathrm{m}^{2}$ \\
\hline Number of Nodes & $N=16,100,256$ \\
\hline Transmission Range & $r_{0}=d$ \\
\hline \hline \multicolumn{2}{|c|}{ Random } \\
\hline Density(nodes/m $\left.{ }^{2}\right)$ & $\rho \in\left\{25 \cdot 10^{-6}, 2 \cdot 10^{-4}\right\}$ \\
\hline Transmission Range $(\mathrm{m})$ & $r_{0}=180$ \\
\hline
\end{tabular}

the generic simulated flocking creatures boids. The basic flocking model consists of three simple steering behaviours which describe how an individual boids moves based on the positions and velocities of its nearby flockmates (see Fig. 7).

Each boids has direct access to the whole scene's geometric description, but flocking requires that it reacts only to flockmates within a certain small neighborhood around itself. The neighborhood is characterized by a distance (measured from the center of the boids) and an angle, measured from the boids direction of flight. Flockmates outside this local neighborhood are ignored. The neighborhood could be considered a model of limited perception (as by fish in murky water) but it is probably more correct to think of it as defining the region in which flockmates influence a boids steering.

\section{Simulation Results}

In this section, we present the simulation results of our proposed WSN. We simulated the network by means of NS2 simulator, with the support of NRL libraries.

In Tables I and II, we summarise the values of parameters used in our WSN. Let us note that the power values concern the power required to transmit and receive one bit, respectively. They do not refer to the radiated power at all. This is also the energy model implemented in the widely used NS-2 simulator.

\section{A. Performance Metrics}

In this paper, we evaluated the performance of the proposed model with two performance metrics: goodput and depletion. The goodput is defined at the sink, and it is the 
Table II

RADIO MODEL AND SYSTEM PARAMETERS.

\begin{tabular}{|c|c|}
\hline \multicolumn{2}{|c|}{ Radio Model Parameters } \\
\hline Path Loss Coefficient & $\alpha=2.7$ \\
\hline Variance & $\sigma_{\mathrm{dB}}^{2}=16 \mathrm{~dB}$ \\
\hline Carrier Frequency & $916 \mathrm{MHz}$ \\
\hline Antenna & omni \\
\hline Threshold (Sensitivity) & $\gamma=-118 \mathrm{~dB}$ \\
\hline \multicolumn{2}{|c|}{ Other Parameters } \\
\hline Reporting Frequency & $T_{r}=[0.1,1000] \mathrm{pps}^{1}$ \\
\hline Interface Queue Size & 50 packets \\
\hline UDP Packet Size & 100 bytes \\
\hline Detection Interval $\tau$ & $30 \mathrm{~s}$ \\
\hline
\end{tabular}

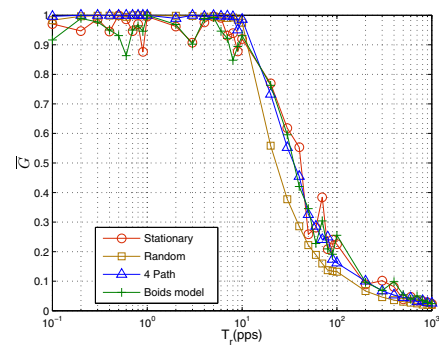

Figure 8. Average goodput for 16 nodes.

received packet rate divided by the sent packets rate. Thus:

$$
G(\tau)=\frac{N_{r}(\tau)}{N_{s}(\tau)}
$$

where $N_{r}(\tau)$ is the number of received packet at the sink, and the $N_{s}(\tau)$ is the number of packets sent by sensor nodes which detected the phenomenon. Note that the eventreliability is defined as $G_{R}=\frac{N_{r}(\tau)}{R(\tau)}$, where $R$ is the required number of packets or data in a time interval of $\tau$ seconds.

As long as the WSN is being used, a certain amount of energy will be consumed. The energy consumption rate directly affects the life-time of the network, i.e., the time after which the WSN is unusable. The energy depletion is a function of the reporting rate as well as the density of the sensor network. Recall that the density of the network in the event-driven scenario correlates with the number of nodes that report their data. Accordingly, we define the consumed energy by the network in the detection interval $\tau$ as:

$$
\bar{\Delta}(\tau)=\frac{N E_{I}-\sum_{i=1}^{N} e_{i}(\tau)}{N \tau}
$$

where $e_{i}(t)$ is the node energy at time $t$ and the means are computed over the number of nodes. The number of nodes $N$ is set as power of integers in order to analyse the behaviour of the scaled versions of the network.

\section{B. Simulation Results and Discussion}

For AODV routing protocol, the sample averages of Eq. (4) and Eq. (5) are computed over 20 simulation runs, and they are plotted from Fig. 8 to Fig. 13. In these figures, the vertical axis shows the goodput or consumed energy, while, the horizontal axis shows is the reporting frequency. We set

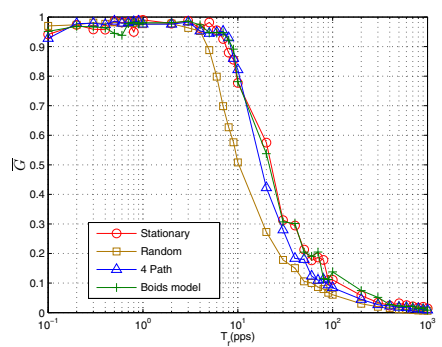

Figure 9. Average goodput for 100 nodes.

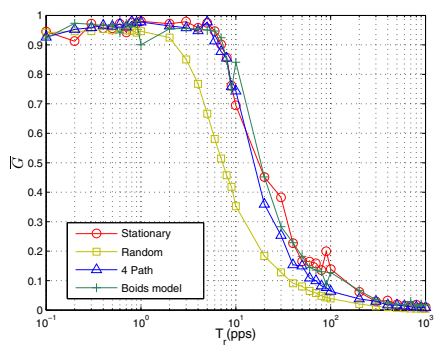

Figure 10. Average goodput for 256 nodes.

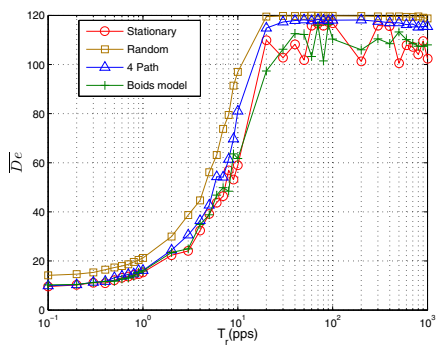

Figure 11. Average depletion for 16 nodes.

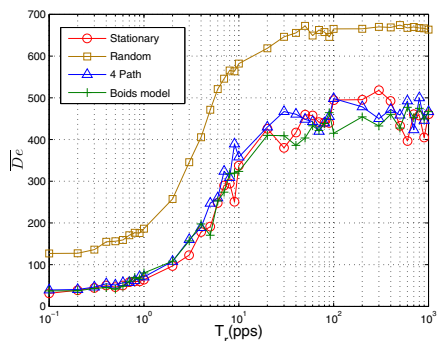

Figure 12. Average depletion for 100 nodes.

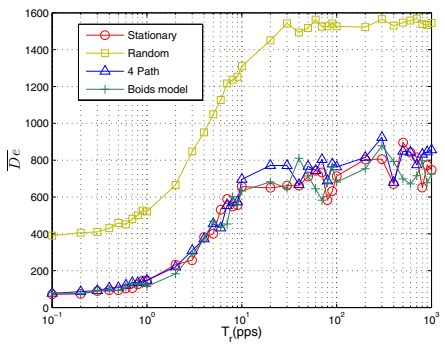

Figure 13. Average depletion for 256 nodes.

the reporting frequency from 0.1 to 1000 . At a particular value of $T_{r}$ ( $\left.\sim 10 \mathrm{pps}\right)$, the goodput decreases abruptly, because the network has reached the maximum capacity.

The goodpout results are plotted from Fig. 8 to Fig. 10. 
As shown in Fig. 8, for the case of 16 nodes, the goodput of stationary event model and boids model are unstable when the $T_{r}$ is lower than $10 \mathrm{pps}$. When the $T_{r}$ is larger than 10 pps, the goodput of random path has the worst performance. When the number of sensor node increase to 100 nodes, as shown in the Fig. 9, the goodput of stationary event is stable. The goodput of random path is lower compared with other cases. Also, the goodput for 100 nodes is lower than 16 nodes. The performance results have the same trend also for 256 nodes as shown in Fig. 10.

The results of consumed energy (depletion) are shown from Fig. 11 to Fig. 13. The consumed energy increases when the number of nodes is increased. In the case of 16 nodes, all the models perform the same, but the performance of random is not good. When the number of nodes increases from 100 to 256, the consumed energy of random path increases much more than the others models. The explanation of this effect is not simple, because it is intermingled with the dynamics of MAC and routing protocol. We also found that the consumed energy of boids model is the lowest in four cases. This show that the event movement with boids model can decrease the consumed energy in the large scale WSNs.

\section{CONCLusions}

In this paper, we presented our simulation results of WSN for four scenarios of event movement. We used the goodput and consumed energy metrics to measure the performance.

From the simulation results, we conclude as follows:

- For the case of 16 nodes, the goodput of stationary event model and boids model are unstable when the $T_{r}$ is lower than 10 pps.

- When the $T_{r}$ is larger than $10 \mathrm{pps}$, the goodput of random path has the worst performance.

- When the number of sensor node increase to 100 nodes, the goodput of stationary event is stable.

- The goodput of random path is lower compared with other cases. Also, the goodput for 100 nodes is lower than 16 nodes. The performance results have the same trend also for 256 nodes

- The consumed energy increases when the number of nodes is increased.

- In the case of 16 nodes, all the models perform the same, but the performance of random is not good.

- When the number of nodes increases from 100 to 256 , the consumed energy of random path increases much more than the others models.

- The consumed energy of boids model is the lowest in four cases. This show that the event movement with boids model can decrease the consumed energy in the large scale WSNs.

In the future, we would like to increase the number of event nodes because the boids model can be applied for many event nodes. We also would like to consider the case of other routing and MAC protocols. We plan to evaluate the performance of WSNs for other metrics such as pathloss and routing efficiency.

\section{ACKNOWLEDGEMENT}

The authors would like to thank KDDI Foundation and Japanese Society for the Promotion of Science (JSPS) for supporting this work.

\section{REFERENCES}

[1] I. F. Akyildiz and I. H. Kasimoglu, "Wireless Sensor and Actor Networks: Research Challenges", Ad Hoc Networks Journal (Elsevier), Vol. 2, No. 4, pp. 351-367, October 2004.

[2] O. Younis and S. Fahmy, "HEED: A Hybrid, Energyefficient, Distributed Clustering Approach for Ad-Hoc Sensor Networks", IEEE Transactions on Mobile Computing, Vol. 3, No. 4, pp. 366-379, October 2004.

[3] S. Giordano and C. Rosenberg, "Topics in Ad Hoc and Sensor Networks", IEEE Communication Magazine, Vol. 44, No. 4, pp. 97-97, 2006.

[4] J. N. Al-Karaki and A. E. Kamal, "Routing Techniques in Wireless Sensor Networks: A Survey", IEEE Wireless Communication, Vol. 11, No. 6, pp. 6-28, December 2004.

[5] T. Yang, G. De Marco, M. Ikeda, and L. Barolli, "Impact of Radio Randomness on Performances of Lattice Wireless Sensor Networks based on Event-Reliability Concept", Mobile Information Systems (MIS), Vol.2, No.4, pp. 211-227, 2006.

[6] G. De Marco, T. Yang, and L. Barolli, "Impact of Radio Irregularities on Topology Tradeoffs of WSNs", Proc. of NBiS2006/DEXA-2006 (Krakow, Poland), pp. 50-54, September 2006.

[7] G. W. Allen, K. Lorincz, M. Welsh, O. Marcillo, J. Johnson, M. Ruiz, and J. Lees, "Deploying a Wireless Sensor Network on an Active Volcano", IEEE Internet Computing, Vol. 10, No. 2, pp. 18-25, March/April 2006.

[8] C. Cooper, "A Note on the Connectivity of 2-Regular Digraphs", Random Structures Algorithms, Vol. 4, No. 4, pp. 469-472, 1993.

[9] Network Simulator, http://www.isi.edu/nsnam/ns/.

[10] Crossbow Technology Inc., http://www.xbow.com/.

[11] T.S. Rappaport, "Wireless Communications", Prentice Hall PTR, 2001.

[12] G. Zhou, T. He, S. Krishnamurthy, and J. A. Stankovic, "Models and Solutions for Radio Irregularity in Wireless Sensor Networks", ACM Transaction on Sensors Network, Vol. 2, No. 2, pp. 221-262, May 2006.

[13] W. Ye, J. Heidemann, and D. Estrin, "Medium Access Control with Coordinated Adaptive Sleeping for Wireless Sensor Networks", IEEE/ACM Transaction Networking, Vol.12, No. 3, pp. 493-506, June 2004.

[14] C. Perkins (Editor), "Ad Hoc Networks", Addison-Wesley, 2001.

[15] Özgür B. Akan and I. F. Akyildiz, "Event-to-sink Reliable Transport in Wireless Sensor Networks", IEEE/ACM Transactions on Networking, Vol. 13, No. 5, pp. 1003-1016, October 2005.

[16] D. Tacconi, I. Carreras, D. Miorandi, A. Casile, F. Chiti, and R. Fantacci, "A System Architecture Supporting Mobile Applications in Disconnected Sensor Networks", IEEE Globecom 2007, Washington, D.C, USA, pp. 833-837, November 2007. 